\title{
Bone induction: regeneration through chaos
}

\author{
Roland Manfred Klar* \\ Bone Research Laboratory, Medical Research Council/University of the Witwatersrand, Johannesburg, South Africa \\ *Correspondence: roland.klar@students.wits.ac.za
}

\section{REGENERATION THROUGH CHAOS}

Ever since Hippocrates (400BC) reported on the unique phenomenon of bone healing without apparent scarring, biological scientists have been trying to unravel the unique and primary controlling mechanism by which bone heals itself with the principle of replicating and controlling its regeneration.

For centuries surgeons have tried to restore large bony defects with little or no success, until the publication of bone regeneration studies by Schede (in Senn, 1889), who utilized blood coagulation as a method to regenerate osteogenic defects. Senn (1889) then developed a medical treatment for osteogenic repair based on Schede's blood coagulation principle in canine models, in which decalcified bone matrix was used as a means to repair bone defects. Despite these discoveries, bone grafting, a treatment still utilized to repair minor areas of bone defects replaced these techniques since grafted bone was able to regenerate faster. However, the limitations of bone grafting were and are still that only minor defects can be regenerated as the amount of harvested bone for repairing larger defects can endanger a patient's health. The need to develop synthetic biomaterials, such as the biomimetic matrices, derived from coral, comprised of calcium phosphate thus resembling vertebral bone, as developed by Weber et al. (1971), provided a structural alternative to bone grafting.

Biomaterials have to be of a specific composition with a specific ratio of calcium phosphate/hydroxyapatite, and have a pore size that facilitates cellular migration and attachment. Yet, the greatest problem is that on their own, macroporous devices only regenerate bone very slowly compared to normal autografts.

Research has thus focused on understanding what it is that modulates bone regeneration on a molecular level with the goal of controlling the modulators and thus the rate of regeneration.
Bone morphogenetic proteins (BMPs) firstly hypothesized by Urist (1965) later extracted (Wang et al., 1988) and cloned by Wozney et al. (1988) from native bone, were amongst first signaling morphogens implicated in the stimulation of bone regeneration, when these successfully induced bone formation. Later transforming growth factor- $\beta$ family members, particularly the three mammalian TGF- $\beta$ isoforms were also shown to be osteoinductive when implanted into non-human primates Papio ursinus (Ripamonti et al., 2010). With the development of assays stimulating a cellular response by applying specific directional forces (like gravity), coupled with changes in device geometry, which act as non-biological signaling stimuli, Ripamonti (2004), Nelson et al. (2005), and Ingber (2005), provided further evidence that bone induction, and its subsequent regeneration, is not restricted to biological modulation. Thus the environment is also a crucial factor involved in the induction and subsequent regeneration of bone. Yet the question remains as to which signaling pathway out of this apparent chaotic molecular scenario is the primordial stimulus required to successfully replicate results achieved by autologous bone grafting?
The answer is perhaps more apparent than what is generally believed. An experiment conducted by Ripamonti et al. (1997), provided a new concept, which moved away from studying the effects of single stimuli and how these effected bone formation. Loading a biomimetic matrix with more than one morphogen, osteogenic protein-1 (OP- 1 ) and TGF- $\beta_{1}$, and then implanting these into heterotopic (non-bony) sites of $P$. ursinus showed a near twofold increase in the amount of bone formed as compared to any previous bone regeneration experiments (Ripamonti et al., 2010). Though it could be argued that the yield of bone formation by induction through the usage of these two morphogens is still lower than that of a bone graft, the findings may have inadvertently provided a possible solution for improving the stimulation of bone induction into macroporous devices, when the molecular analytical results are taken into account.

Even though the synergy is more successful in inducing bone formation than either the scaffold solo or with each morphogen implanted singly, the molecular data revealed that OP-1 and TGF- $\beta_{3}$ expression is directly dependant on each other (Ripamonti, 2004). Experiments in

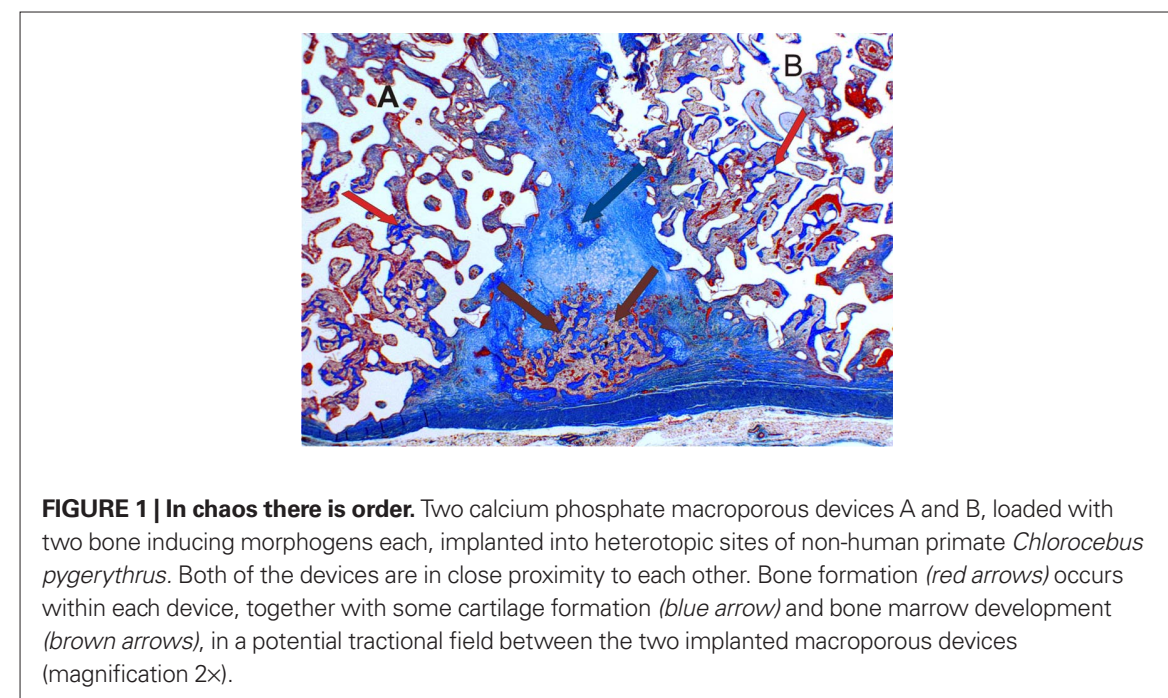


which zoledronic acid a bisphosphonate derivative, prevented the active binding of osteoclasts to the macroporous devices (Ripamonti et al., 2010), demonstrated the loss of the expression of either OP-1 or TGF- $\beta_{3}$ morphogens and their subsequent signaling protein secretion. This indicates that both morphogens must be expressed in order to facilitate successful bone formation.

Thus both osteoclast attachment (driven by the chemical composition and physical characteristics of the scaffold) and the subsequent expression of these two morphogens are required to stimulate proper bone formation by autoinduction. Furthermore this suggests that the primordial initiating mechanism is not comprised of a singular all-controlling pathway, but of a mixture of more than one signal (Figure 1). With this knowledge the author is of the opinion that all future bone induction research should focus on finding and analyzing which mixtures of stimulatory, regulatory/ inhibitory morphogens coupled with other non-biological signaling mechanisms work best together to increase the rate and the deposition of bone. After all in chaos is there not order? (Lorenz, 1963).

\section{REFERENCES}

Ingber, D. E. (2005). Mechanical control of tissue growth: function follows form. Proc. Natl. Acad. Sci. U.S.A. 102, 11571-11572.

Lorenz, E. N. (1963). Deterministic nonperiodic flow. J. Atmos. Sci. 20, 130-141.

Nelson, C. M., Jean, R. P., Tan, J. L., Liu, W. F., Sniadecki, N. J., Spector, A. A., and Chen, C. S. (2005). Emergent patterns of growth controlled by multicellular form and mechanics. Proc. Natl. Acad. Sci. U.S.A. 102, 11594-11599.

Ripamonti, U. (2004). Soluble, insoluble and geometric signals sculpt the architecture of mineralized tissues. J. Cell. Mol. Med. 8, 169-180.

Ripamonti, U., Duneas, N., van den Heever, B., Bosch, C., and Crooks, J. (1997). Recombinant transforming growth factor- $\beta 1$ induces endochondral bone in baboon and synergizes with recombinant osteogenic protein-1 (bone morphogenic protein-7) to initiate rapid bone formation. J. Bone Miner. Res. 2, 1584-1595.

Ripamonti, U., Klar, R. M., Renton, L. F., and Ferretti, C. (2010).Synergisticinduction of bone formation by hOP1 , hTGF- $\beta_{3}$ and inhibition by zoledronate in macroporous coral-derived hydroxyapatites. Biomaterials 1, 1-11.
Senn, N. (1889). On the healing of aseptic bone cavities by implantation of antiseptic decalcified bone. Am J. Med. Sci. 98, 219-243.

Urist, M. R. (1965). Bone: formation by autoinduction. Science 150, 893-899.

Wang, E. A., Rosen, V., Cordes, P., Hewick, R. M., Kriz, M. J., Luxenberg, D. P., Sibley, B. S., and Wozney, J. M. (1988). Purification and characterization of other distinct bone-inducing factors. Proc. Natl. Acad. Sci. U.S.A. 85, 9484-9488.

Weber, J. N., White, E. W., and Lebiedzik, J. (1971). New porous biomaterials by replication of echinoderm skeletal microstructures. Nature 233, 337.

Wozney, J. M., Rosen, V., Celeste, A. J., Mitsock, L. M., Whitters, M. J., Kriz, R.W., Hewick, R. M., and Wang, E. A. (1988). Novel regulators of bone formation: molecular clones and activities. Science 242, 1528-1534.

Received:04 April 2011; accepted:29April 2011; published online: 31 May 2011.

Citation: Klar RM (2011) Bone induction: regeneration through chaos. Front. Physio. 2:21. doi: 10.3389/ fphys.2011.00021

This article was submitted to Frontiers in Systems Biology, a specialty of Frontiers in Physiology.

Copyright $(0) 2011$ Klar. This is an open-access article subject to a non-exclusive license between the authors and Frontiers Media SA, which permits use, distribution and reproduction in other forums, provided the original authors and source are credited and other Frontiers conditions are complied with. 